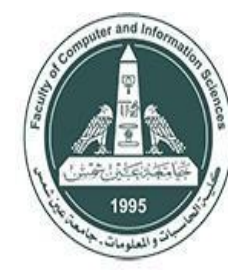

International Journal of Intelligent Computing and Information Sciences

https://ijicis.journals.ekb.eg/

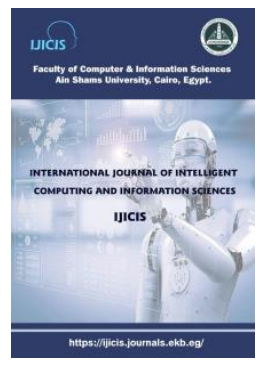

\title{
A Review of Leveraging Blockchain based Framework Landscape in Healthcare Systems
}

\begin{tabular}{l} 
Mohammed K. Elghoul * \\
Scientific Computing \\
Department, \\
Faculty of Computer and \\
Information Sciences, \\
Ain-Shams University \\
mohammed.elghoul@ cis.asu.e \\
\multicolumn{1}{c}{ du.eg }
\end{tabular}

Sayed F. Bahgat

Scientific Computing

Department,

Faculty of Computer and Information

Sciences,

Ain-Shams University

sayedfadel@cis.asu.edu

.eg
Ashraf S. Hussein

Scientific Computing Department,

Faculty of Computer and Information Sciences,

Ain-Shams University

ashussein@cis.asu.edu.eg
Safwat H. Hamad

Scientific Computing

Department, Faculty of Computer and Information Sciences, Ain-Shams University shamad@cis.asu.edu.eg

Received 2021-05-25; Revised 2021-9-18; Accepted 2021-9-18

\section{Abstract}

Blockchain is a technology new technology implemented for the first time in 2008 for the bitcoin cryptocurrency [1]. Blockchain technology is an innovative, distributed, and immutable ledger technology providing a decentralized management solution for transaction data [2].

The purpose of this article was to review the growth of the use of blockchain technology in the healthcare data. In this article, an analysis of the existing blockchain technology research and findings in the health care domain was conducted. The goal was to find relevant implementation of the blockchain technology in the healthcare domain and highlight the challenges and potential methodologies. This article covers an overarching introduction and Background about blockchain in the healthcare domain. Furthermore, the research methodology, an analysis of the information and the results found. The results show that Blockchain still has many challenges such as scalability and security problem [3], however the usage of blockchain is increasing in different scientific research areas [4-7] in general and in healthcare area in particular is growing exponentially [8].

\footnotetext{
* Corresponding author: Mohammed K. Elghoul

Scientific Computing Department, Faculty of Computer and Information Sciences, Ain-Shams University

E-mail address: mohammed.elghoul@ @is.asu.edu.eg
} 


\section{Introduction}

Health information technology (HIT) systems play a substantial role in everybody's life. Medical data, in the form of electronic medical record, is the main asset for those systems. However, those systems have several challenges due to the growth of the amount of medical data gathered electronically, the sensitivity of patient's data, and the need to allow data exchange of patient's health records between the multiples systems used by healthcare professionals. Therefore, it is challenging to store the data in a traditional centralized data warehouse. It is substantial to ensure that the data integrity is safeguarded, easily accessible, and secured [9].

Given the challenges in the current HIT systems, there is a need to foster a new solution to healthcare data management. The new healthcare data management system should be able to fulfill several objectives, including (a) medical records data must be protected against unauthorized use; (b) create trust among healthcare stockholders through data sharing that's patient-centric and transparent; (c) a distributed solution to overcome the limitation of centralized systems; (d) finally, the system should provide a mechanism for data authenticity in order to verify the integrity of medical records data [1].

The blockchain technology is an innovative, distributed, and immutable ledger technology which providing a decentralized management solution for data transaction. The Bitcoin cryptocurrency was the first implementation of blockchain technology in 2008. The blockchain technology has its challenges around security, privacy, and scalability. However, it has a great potential to solve various problems in a distributed environment, Blockchain technology is applicable not only in the financial transaction systems, but it is transforming our society, as the digital footprint we leave behind through our use of cars, smartphones, healthcare, vote, and even personal identification increases in breadth and depth.

The natural properties of the blockchain technology can be used to realize the objectives mentioned previously: (a) permissioned blockchain networks enable granular access control for medical records can be achieved through, supporting granular- level access mechanisms such as channels; (b) blockchainsupported smart contracts enables patient-centric and transparent data sharing and control; (c) the blockchain distributed consensus mechanism overcomes the limitation of centralization; (d) and, a blockchain is immutable preserving the integrity of data; thus the integrity of the data saved on a blockchain is verifiable and provable.

Blockchain technology could improve information security, data decentralization, search \& data retrieval, and data integrity in the healthcare industry. However, the first evolution of blockchain technology was designed mainly for cryptocurrency transactions with no focus to be extended or even applied to other industries such as the health care industry. Currently, there is an emerging effort to use general-purpose blockchain technology to develop healthcare systems. The biggest challenge is being phased is there is no consensus on which blockchain framework is most suitable for developing healthcare applications. 


\section{Background of Blockchain}

Blockchain technology is a distributed ledger that shares data between a network of peers [13]. Bitcoin cryptocurrency was the first implementation of Blockchain technology in 2008 [1,2]. The main advantage of blockchain technology is lower cost, faster, and more efficient and secured data sharing through the ability to connect distributed network nodes directly without dependency on any trusted third party.

\subsection{Key characteristics of Blockchain}

Blockchain technology has four key characteristics - decentralization, immutability, audit \& traceability, and data integrity [3]. Blockchain technology has no dependency on a central authority to control the data that gets sent to it, because blockchain technology uses various consensus algorithms that decide whether to agree on the data is being sent or not in a peer-to-peer network. Record immutability is another inherent characteristic in blockchain technology because it's impossible to remove a record after it's accepted and stored onto a blockchain because it's stored across multiple nodes in the distributed ledger. Lineages is a supported feature because new blooks get linked to previous blocks by including the hash of the previous one so a chain of blooks is formed. In a blockchain every transaction is verified to the known root which enabling the integrity of the data in a blockchain to be verifiable because the tree structure that's formed in Merkle tree [10].

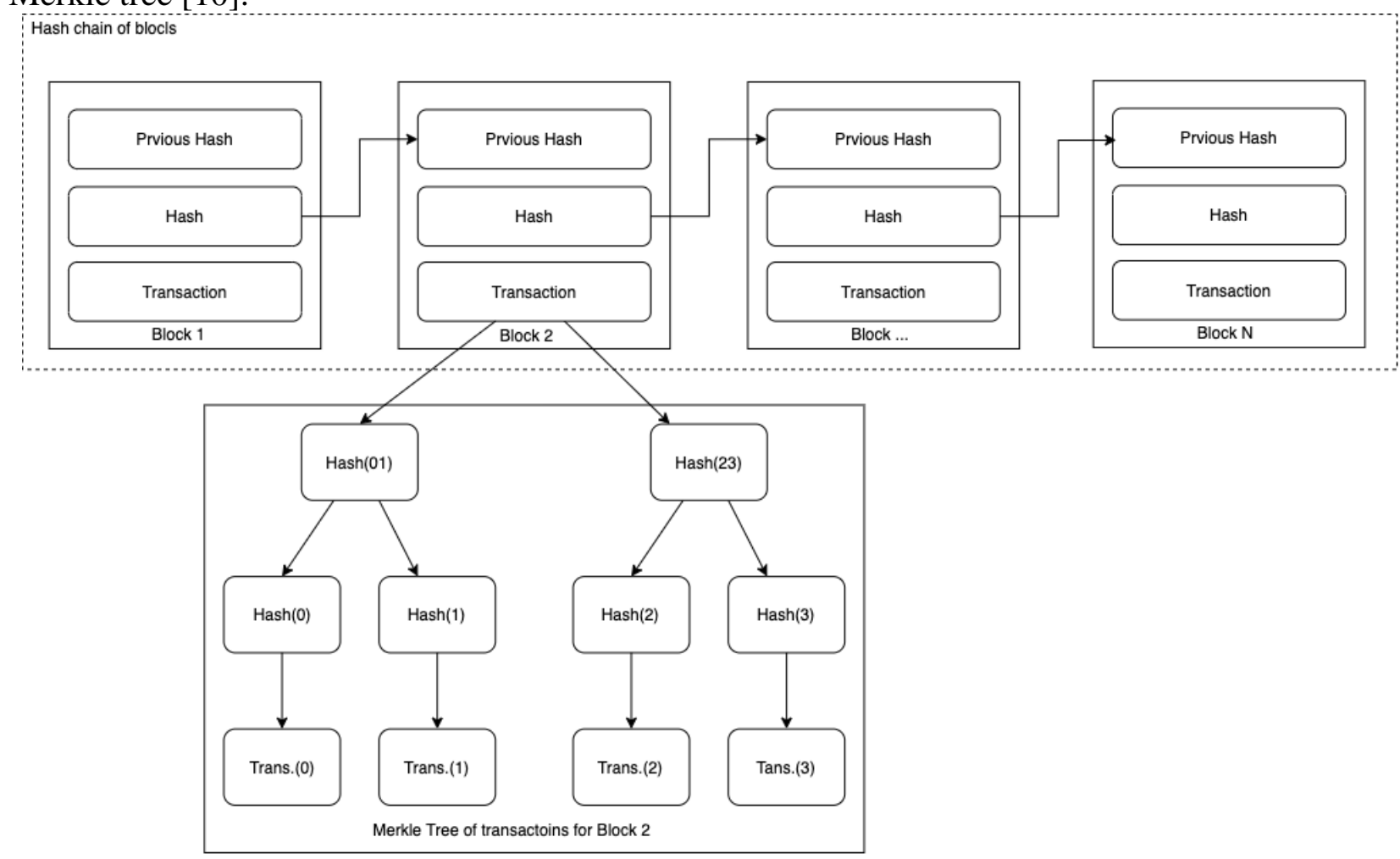

Figure:1 Blockchain record structure and Merkle Tree structure [8] 


\subsection{Types of blockchain}

Blockchains can be of three different types: public, consortium and private [3]. The first type is public blockchains where it can be accessed and viewed by any user who can join and contribute to the consensus protocol [8]. Cryptocurrencies is the major application field for this type of blockchain. Bitcoin and Ethereum are examples of public/ permission-less chains. The second type of blockchain is consortium which is a partially centralized, where only selected group of users have permissions to view and contribute to the consensus protocol. Finally, private blockchain is distributed in a decentralized manner through the network. One center authority manages the selected nodes that participate in the network [3]. Due to the diversity of the applications and domains that can leverage the blockchain technology, there is no consensus of which distributing qualities and consensus mechanisms are required to label a technology as "blockchain". In Table 1 below a comparison of the three different types of block chains is shown.

\section{Table 1}

Type of blockchains overview [3]

\begin{tabular}{|l|l|l|l|}
\hline Property & Public blockchain & Consortium blockchain & Private blockchain \\
\hline Consensus determination & All miners & Selected set of nodes & One organization \\
\hline Read permission & Public & Public or restricted & Public or restricted \\
\hline Immutability & Nearly impossible & Could be tampered & Could be tampered \\
\hline Efficiency & Low & High & High \\
\hline Centralized & No & Partial & Yes \\
\hline Consensus process & Permissionless & Permissioned & Permissioned \\
\hline
\end{tabular}

There are multiple blockchain frameworks and platforms that can be used or combined together to deliver decentralized systems. The most popular ones are Ethereum [11] and Hyperledger [12] which can be used to develop new systems or create new protocols.

\subsection{Consensus Protocols}

To keep the transaction and the ledger whole in the blockchain network, all peers must agree on a particular state of the distributed ledger and agree on how to pack the data into blocks. This capability is called a distributed consensus protocol. It is responsible for validating the chronological order of the generated transactions [12], ensuring that all pears in the network come to an agreement on an accurate state of the shared ledger, and responsible for adding the new transactions "blocks" are added to the ledger [13].

There are multiple protocols and the mostly commonly used are Proof-of-Work (PoW), Proof-of-Stack (PoS) and Practical Byzantine Fault Tolerance (PBFT). The major differences between the distributed consensus protocols are round how a node is managed and secured, the energy consumptions, and how the power of adversary is tolerated. 
The PoW protocol is considered the most popular consensus protocol because it is used by Bitcoin. It uses high computing power to solve the hard computational puzzle of finding the selected nodes. The node that computes the hash is rewarded by being selected. The major disadvantage of this protocol is the high energy consumption of large blockchain.

The PoS protocol uses a different technique to select the approved node by determining the stack of each node in a blockchain. The stack is represented by the value or the asset of the node. In the cryptocurrency, the stack is represented by the balance of that currency. Because each node can have a different value this protocol is considered unfair. The nodes with higher assets will have higher advantage than the ones with less assets. Its energy consumption is less compared to the PoW protocol which shows that it is better and supports the move by the Ethereum algorithm to use PoS instead of PoW [3].

PBFT is another consensus protocol and is based on the Byzantine agreement protocol [8]. It is a permissioned protocol, and all nodes must be known to the network. This is considered a limitation when it's used in a public blockchain. Each node in the network preforms three steps. Those steps are preprepared, prepared and commit. It requires two thirds of the votes from all nodes in order to complete all the three steps. The energy consumption is less compared to the previous protocols and it is used in the Hyperledger Fabric [12].

\subsection{The Need for Blockchain in Healthcare}

Blockchain technology usage in healthcare has a great perennial to solve many of the challenge's healthcare domain has. Due to the growth of data in HIT and the need to make the data available via a secure manner, blockchain technology can be used to meet these requirements. Starting from data management and the accuracy of the health records, the blockchain technology can play a pivotal role in various areas such as drug prescriptions, pregnancy, supply chain management, health record management, and any risk data management as well as to support better access control, data sharing and managing of an audit trail of medical activities [13]. Blockchain technology can improve medical data availability, data security, data sharing, traceability and record immutability.

Medical image sharing is another area in the healthcare domain that can take advantage of blockchain technology [14]. Transforming and sharing medical images from physical copies to digital imaging has a significant impact in improving the security and availability of the medical images between providers. The classic way of sharing medical images is for the patients themselves to keep a physical copy on a disk and share it between providers. This solution has the risk of loss of, or damage to the physical copy. Currently, there is a solution called Image Share Network (ISN) that has been developed by Radiological Society of North America (RSNA) [14]. In this solution the images get uploaded to a clearinghouse and persisted In addition to that, a cryptographic hash of a secret token is generated to get the images indexed, for 30 days. During this period, the patient may permit his personal health record vendor to download the images so they can be accessed by healthcare providers.

Although this solution solves the sharing problem in a digital way instead of a physical media transfer, it still has few drawbacks such as centralization that is a single point of failure in addition to having a third 
party "clearinghouse" in the middle between the image centers and Personal Health Records (PHR) as shown the in the architecture diagram below in Figure 2. The following statistics were provided in 2019 [14]

- About a dozen or less of radiology centers are enrolling patients in the network,

- As of 2019, there are four PHR vendors are authorized to retrieve ISN images,

- the Clearinghouse operator is one of the four PHRs,

- About 30 percent of the patients' images was downloaded by the approved PHRs at least once.

This solution is considered a major milestone introducing the concept of a decentralized architecture and as an option that may decrease the barriers to entry and encourage more wider adoption of the image sharing network.

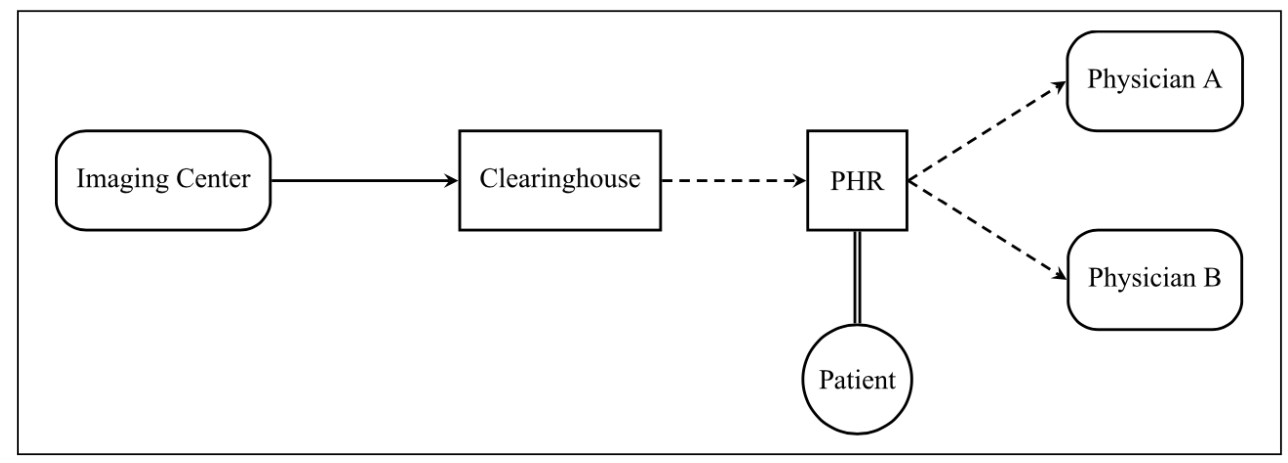

Figure 2. The RSNA Image Share network [14]

\section{Research methodology/ approach}

The research was conducted in various bibliographic databases and it was limited to the healthcare domain.

The research included the following eight electronic sources Google Scholar, Science Direct, IEEE, PubMed, Scopus, Springer SpringerLink, Elsevier Science Direct and ACM.

\section{Analysis and Discussion of Blockchain Technology usage in Health Care}

First, different publications were chosen and analyzed to find out which ones are the most relevant ones in this area. Blockchain technology is exponentially being used in the health care domain specially to manage electronic health records and personal health records. This article identified that there are three potential areas of improvement Access control, Interoperability, and Provenance Data integrity [3]. This found also that Ethereum and Hyperledger fabric are the most commonly used framework in this domain. EHR and PHR are the two highly impacted areas by Blockchain technology with $43 \%$ and $15 \%$ among other areas based on that research and the other areas are covered by about $1 \%$ each. 
The study conducted by Anton [3] showed the usage of the different platforms/frameworks with highest distribution for new blockchain framework 36\% (14) then $28 \%$ (11) for the Ethereum and 10\% (4) for Hyperledger Fabric (Figure 3).

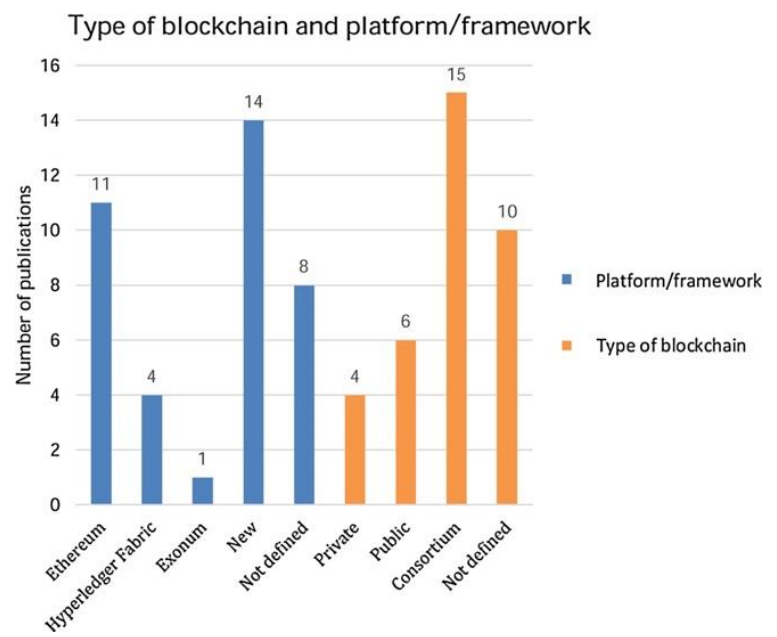

Figure 3. Type of blockchain and platform/framework [3].

Other research conducted in June 2018 identified only publications published after 2015 and is relevant and suitable for this survey. This analysis indicated that blockchain technology is a new technology that can be used in the healthcare domain. Out of 6,375 publications, this article identified 33 publications which are relevant to the use of blackchin technology in the healthcare domain. The 33 publications are divided into 28 proposing solitons that can be implemented and only 5 presenting implementations [13].

This previous research identified the usage of the blockchain platform as the following that 10 publications use Ethereum, 5 publications use Hyperledger Fabric, 1 publication uses Gcoin and 15 publication do not have a defined platform. Data sharing and health records the most areas of application using blockchain with 20 and 18 publication respectively. Access control is another area of application with 15 publication and other smaller numbers for audit trail and supply chain with 6 and 2 publications respectively.

One of the publications focused on showing the number of previously published papers based on the publication types and another representation by the number of papers per proposed versus implemented solution [13]. 


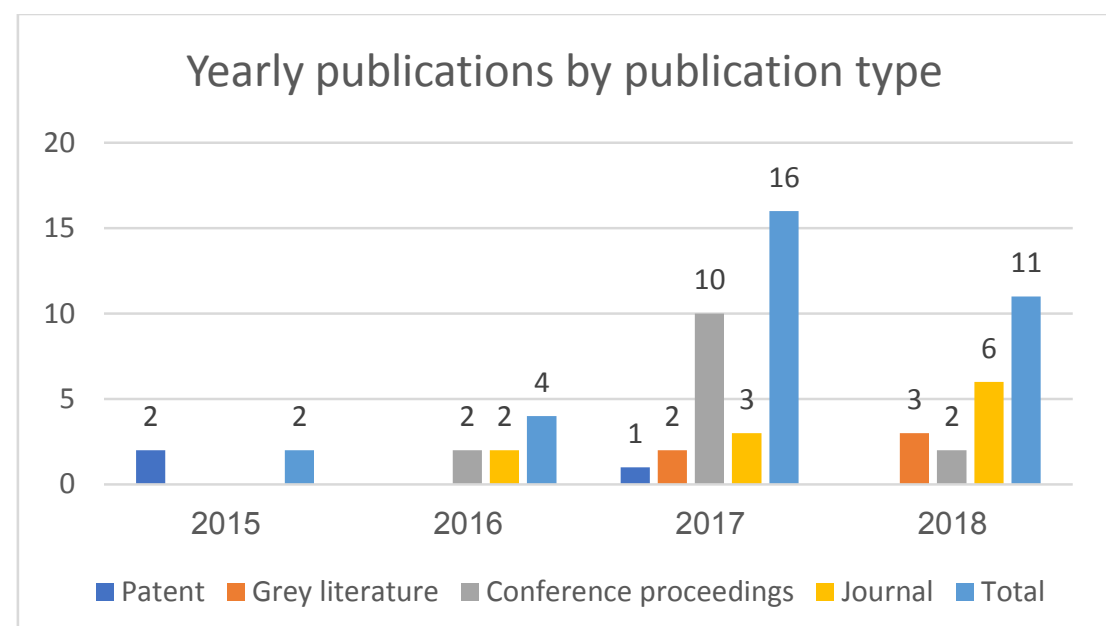

Figure 4. Yearly publications by publication type [13].

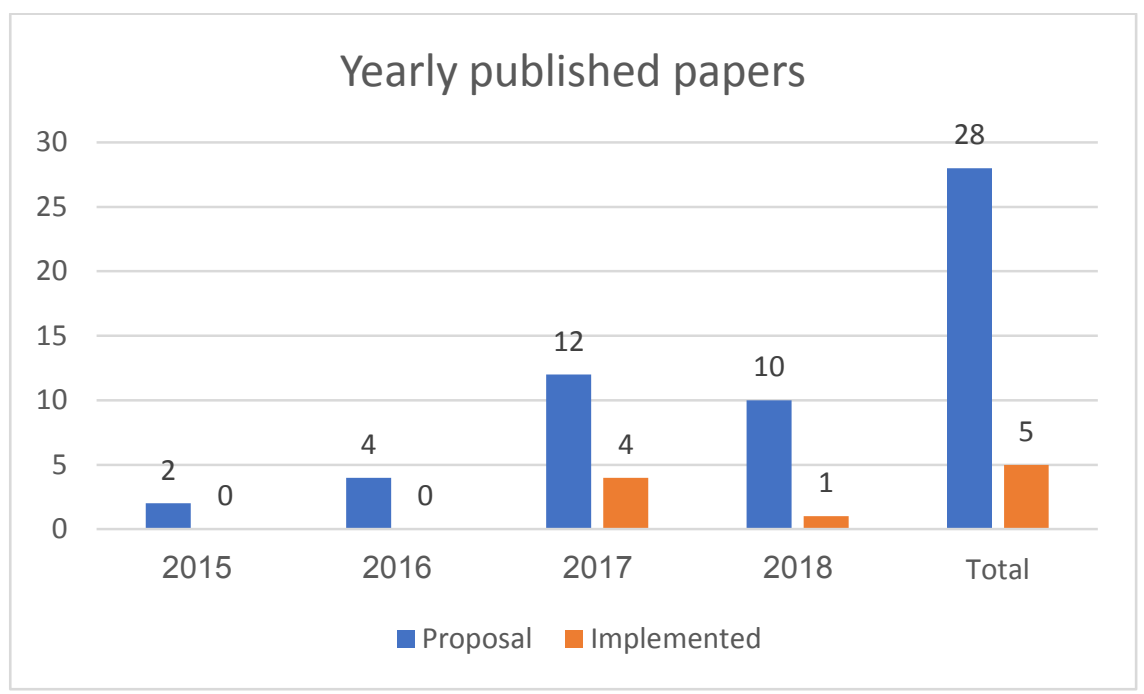

Figure 5. Yearly published papers [13].

The analysis shows that leveraging blockchain technology in the healthcare is a hot topic in the academic and it's in early stage. The current number of approaches and proposed solutions is growing exponentially [3]. In addition to that, the study shows that the average quality score per year of the papers is also increasing (Figure 6). This analysis shows how the blockchain smart contracts can be used on the Ethanium platform and how it can be organized as a consortium blockchain. The analysis shows that most of the solutions are either implemented in a controlled system, personal records systems or in the health care field. This analysis proves that the grows of blockchain in healthcare has the same growth in other academic areas. 


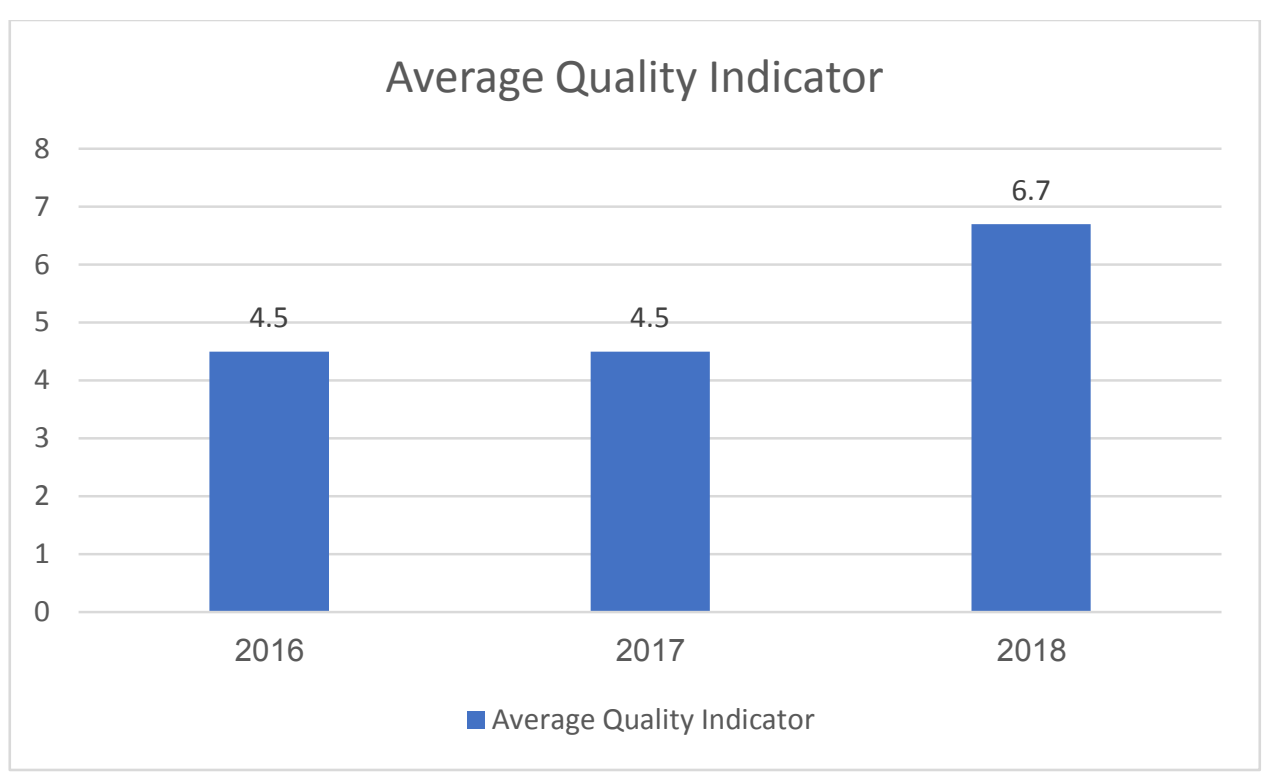

Figure 6. Average quality score per year [3].

In 2019 Asma Khatoon [15] published an article about using blockchain-based smart contract system for health care management. In that article, Asma conducted a review of the blockchain technology usage in health care between 2016 and 2019 [16-40]. Asma implemented a smart contract-based healthcare management system using blockchain technologies that showed how to apply the decentralization priceable within the medical ecosystem. In additional to applying blockchain principles, other benefits are shown as well such as reduced the transaction costs, reduced the administrative burdens, and removed intermediaries.

\section{Conclusion}

The research shows that leveraging blockchain technology in the healthcare domain is trending upwards and that indicates that is also proportionally growing in the academic field. The results shows that the quality of publications has increased as well. Blockchain technology is a decentralized network and published research shows that there is a huge potential for the use in the healthcare domain. We have identified the current status and the different areas in healthcare blockchain can be applied. All results shows that blockchain technology is generally applied in solving data sharing, health record management, access control, lineage and audit trail.

There is a gap between the availability of blockchain elements technical details e.g., platform, algorithms type or the usage of smart contracts and the current publications about framework, architecture or model using blockchain technology in healthcare. Smart contracts in particular could be more used as they enable the automation of processes within a blockchain platform. Most research should have provided either a prototype implementation or even discuss some implementation details of their proposals. 
Most of the implemented solutions and research showed that blockchain technology has a great potential in solving many of the challenges and issues in the current healthcare systems. These issues can be data security, data privacy, legacy network inconsistency, unstructured data collection difficulties, prohibitively high administrative costs.

\section{References}

[1] Agbo, CC, Mahmoud, QH. "Comparison of blockchain frameworks for healthcare applications." Internet Technology Letters. 2019; 2:e122. https://doi.org/10.1002/itl2.122

[2] Nakamoto S., "Bitcoin: a peer-to-peer electronic cash system". 2008. [Online]. https://bitcoin.org/bitcoin.pdf. Accessed March 30, 2019.

[3] Z. Zheng, S. Xie, H. Dai, X. Chen and H. Wang, "An Overview of Blockchain Technology: Architecture, Consensus, and Future Trends," 2017 IEEE International Congress on Big Data (BigData Congress), Honolulu, HI, 2017, pp. 557-564, doi: 10.1109/BigDataCongress.2017.85.

[4] L. Kan, Y. Wei, A. Hafiz Muhammad, W. Siyuan, L. C. Gao and H. Kai, "A Multiple Blockchains Architecture on Inter-Blockchain Communication," 2018 IEEE International Conference on Software Quality, Reliability and Security Companion (QRS-C), Lisbon, Portugal, 2018, pp. 139145, doi: 10.1109/QRS-C.2018.00037.

[5] D. Miller, "Blockchain and the Internet of Things in the Industrial Sector," in IT Professional, vol. 20, no. 3, pp. 15-18, May./Jun. 2018, doi: 10.1109/MITP.2018.032501742.

[6] J. Fiaidhi, S. Mohammed and S. Mohammed, "EDI with Blockchain as an Enabler for Extreme Automation," in IT Professional, vol. 20, no. 4, pp. 66-72, Jul./Aug. 2018, doi: 10.1109/MITP.2018.043141671.

[7] M. Samaniego, U. Jamsrandorj and R. Deters, "Blockchain as a Service for IoT," 2016 IEEE International Conference on Internet of Things (iThings) and IEEE Green Computing and Communications (GreenCom) and IEEE Cyber, Physical and Social Computing (CPSCom) and IEEE Smart Data (SmartData), Chengdu, China, 2016, pp. 433-436, doi: 10.1109/iThingsGreenCom-CPSCom-SmartData.2016.102.

[8] Anton Hasselgren, Katina Kralevska, Danilo Gligoroski, Sindre A. Pedersen, Arild Faxvaag, "Blockchain in healthcare and health sciences-A scoping review," International Journal of Medical Informatics, Volume 134, 2020:104040

[9] T. Benil, J. Jasper, "Cloud based security on outsourcing using blockchain in E-health systems," Computer Networks, vol. no. 178, 2020: 107344

[10] R.C. Merkle (Ed.), "A Certified Digital Signature," Conference on the Theory and Application of Cryptology, Springer, 1989.

[11] Paul J. Taylor, Tooska Dargahi, Ali Dehghantanha, Reza M. Parizi, Kim-Kwang Raymond Choo, "A systematic literature review of blockchain cyber security, " Digital Communications and Networks, Volume 6, Issue 2, 2020: 147-156

[12] Cachin, C.. "Architecture of the Hyperledger Blockchain Fabric," Workshop on Distributed 
Cryptocurrencies and Consensus Ledgers, 2016.

[13] Hölbl, Marko \& Kompara, Marko \& Kamisalic, Aida \& Nemec Zlatolas, Lili. (2018). "A Systematic Review of the Use of Blockchain in Healthcare," Symmetry. 10. 470. 10.3390/sym10100470.

[14] Patel V. "A framework for secure and decentralized sharing of medical imaging data via blockchain consensus," Health Informatics Journal. 2019;25(4):1398-1411. doi: $10.1177 / 1460458218769699$

[15] Khatoon, A. A Blockchain-Based Smart Contract System for Healthcare Management. Electronics 2020, 9, 94. https://doi.org/10.3390/electronics9010094

[16] Daisuke, I.; Kashiyama, M.; Ueno, T. Tamper-resistant mobile health using blockchain technology. JMIR Mhealth Uhealth 2017, 5, e111.

[17] Vazirani, A.A.; O'Donoghue, O.; Brindley, D.; Meinert, E. Implementing Blockchains for Efficient Health Care: Systematic Review. J. Med. Internet Res. 2019, 21, e12439.

[18] S. Rouhani, L. Butterworth, A. D. Simmons, D. G. Humphery and R. Deters, "MediChainTM: A Secure Decentralized Medical Data Asset Management System," 2018 IEEE International Conference on Internet of Things (iThings) and IEEE Green Computing and Communications (GreenCom) and IEEE Cyber, Physical and Social Computing (CPSCom) and IEEE Smart Data (SmartData), Halifax, NS, Canada, 2018, pp. 1533-1538, doi:

10.1109/Cybermatics_2018.2018.00258.

[19] Wu, H.T.; Tsai, C.W. Toward blockchains for health-care systems: Applying the bilinear pairing technology to ensure privacy protection and accuracy in data sharing. IEEE Consum. Electron. Mag. 2018, 7, 65-71.

[20] Shen, B.; Guo, J.; Yang, Y. MedChain: Efficient Healthcare Data Sharing via Blockchain. Appl. Sci. 2019, 9, 1207.

[21] Khezr, S.; Moniruzzaman, M.; Yassine, A.; Benlamri, R. Blockchain technology in healthcare: A comprehensive review and directions for future research. Appl. Sci. 2019, 9, 1736.

[22] Litchfield, A.T.; Khan, A. A Review of Issues in Healthcare Information Management Systems and Blockchain Solutions; CONF-IRM, 2019; Available online: https://aisel.aisnet.org/confirm2019/1/ (accessed on 31 December 2019).

[23] Vora, J.; Nayyar, A.; Tanwar, S.; Tyagi, S.; Kumar, N.; Obaidat, M.S.; Rodrigues, J.J. BHEEM: A Blockchain-Based Framework for Securing Electronic Health Records. In Proceedings of the 2018 IEEE Globecom Workshops (GC Wkshps), Abu Dhabi, UAE, 9-13 December 2018.

[24] Zhang, P.; Schmidt, D.C.; White, J.; Lenz, G. Blockchain Technology Use Cases in Healthcare. In Advances in Computers; Elsevier: Amsterdam, The Netherlands, 2018; Volume 111, pp. 1-41.

[25] Siyal, A.; Junejo, A.; Zawish, M.; Ahmed, K.; Khalil, A.; Soursou, G. Applications of Blockchain Technology in Medicine and Healthcare: Challenges and Future Perspectives. Cryptography 2019, 3, 3 .

[26] Jamil, F.; Hang, L.; Kim, K.; Kim, D. A Novel Medical Blockchain Model for Drug Supply Chain Integrity Management in a Smart Hospital. Electronics 2019, 8, 505.

[27] Lee, S.H.; Yang, C.S. Fingernail analysis management system using microscopy sensor and 
blockchain technology. Int. J. Distrib. Sens. Netw. 2018, 14.

[28] Agbo, C.C.; Mahmoud, Q.H.; Eklund, J.M. Blockchain technology in healthcare: A systematic review. Healthcare 2019, 7, 56.

[29] Azaria, A.; Ekblaw, A.; Vieira, T.; Lippman, A. Medrec: Using Blockchain for Medical Data Access and Permission Management. In Proceedings of the 2016 2nd International Conference on Open and Big Data (OBD), Vienna, Austria, 22-24 August 2016.

[30] N. Nchinda, A. Cameron, K. Retzepi and A. Lippman, "MedRec: A Network for Personal Information Distribution," 2019 International Conference on Computing, Networking and Communications (ICNC), Honolulu, HI, USA, 2019, pp. 637-641, doi: 10.1109/ICCNC.2019.8685631

[31] Zhang, P.; White, J.; Schmidt, D.C.; Lenz, G. Design of Blockchain-Based Apps Using Familiar Software Patterns to Address Interoperability Challenges in Healthcare. In Proceedings of the PLoP-24th Conference on Pattern Languages of Programs, Vancouver, BC, Canada, 22-25 October 2017.

[32] Kumar, T.; Ramani, V.; Ahmad, I.; Braeken, A.; Harjula, E.; Ylianttila, M. Blockchain Utilization in Healthcare: Key Requirements and Challenges. In Proceedings of the 2018 IEEE 20th International Conference on e-Health Networking, Applications and Services (Healthcom), Ostrava, Czech Republic, 17-20 September 2018.

[33] Ali, Saqib et al. "A Blockchain-Based Decentralized Data Storage and Access Framework for PingER." 2018 17th IEEE International Conference On Trust, Security And Privacy In Computing And Communications/ 12th IEEE International Conference On Big Data Science And Engineering (TrustCom/BigDataSE) (2018): 1303-1308

[34] PengCheng Wei, Dahu Wang, Yu Zhao, Sumarga Kumar Sah Tyagi, Neeraj Kumar, "Blockchain data-based cloud data integrity protection mechanism," Future Generation Computer Systems, Volume 102, 2020: 902-911

[35] Krishnapriya S, Greeshma Sarath, "Securing Land Registration using Blockchain," Procedia Computer Science, Volume 171, 2020: 1708-1715

[36] Ahmed Farouk, Amal Alahmadi, Shohini Ghose, Atefeh Mashatan, "Blockchain platform for industrial healthcare: Vision and future opportunities," Computer Communications, Volume 154, 2020: $223-235$

[37] Qinghua Lu, Xiwei Xu, Yue Liu, Ingo Weber, Liming Zhu, Weishan Zhang, "uBaaS: A unified blockchain as a service platform," Future Generation Computer Systems, Volume 101, 2019: 564575

[38] Dasaklis, Thomas \& Casino, Fran \& Patsakis, Costas \& Douligeris, Christos."A framework for supply chain traceability based on blockchain tokens,", 2019

[39] Koteska, Bojana et al. "Blockchain Implementation Quality Challenges: A Literature Review." SQAMIA, 2017

[40] Ingo Weber, Xiwei Xu , Regis Riveret, Guido Governatori, Alexander Ponomarev, Jan Mendling, "Untrusted Business Process Monitoring and Execution Using Blockchain, " 2016, 10.1007/9783-319-45348-4_19 
[41] Dubovitskaya A, Baig F, Xu Z, Shukla R, Zambani P, Swaminathan A, Jahangir M, Chowdhry K, Lachhani R, Idnani N, Schumacher M, Aberer K, Stoller S, Ryu S, Wang F, "ACTION-EHR: Patient-Centric Blockchain-Based Electronic Health Record Data Management for Cancer Care, "J Med Internet Res 2020, 22(8), e13598, URL: https://www.jmir.org/2020/8/e13598

[42] Lemieux, V.L., "Trusting records: is Blockchain technology the answer?", Records Management Journal, 2016, Vol. 26 No. 2, pp. 110-139. https://doi.org/10.1108/RMJ-12-2015-0042 